\title{
Three Node Tandem Communication Network Model with Dynamic Bandwidth Allocation and Non Homogeneous Poisson Arrivals
}

\author{
M.V.Rama Sundari \\ Dept of CSSE \\ Andhra University \\ Visakhapatnam
}

\author{
K.Srinivas Rao \\ Dept of Statistics \\ Andhra University \\ Visakhapatnam
}

\author{
P.Srinivasa Rao \\ Dept of CSSE \\ Andhra University \\ Visakhapatnam
}

\author{
P.Suresh Varma \\ Dept of CS \\ Adikavi Nannaya \\ University \\ Rajahmundry
}

\begin{abstract}
Communication networks create lot of interest due to their ready applicability in performance evaluation of several communication systems. In communication systems it is customary to consider that the arrivals are characterized by Poisson process. This assumption holds good if the arrivals are homogeneous and independent of time. But in many tele and satellite communication systems the arrivals are non homogeneous and the arrival rate is time dependent. Hence, in this paper we develop and analyze a three node communication network model with the assumption that the arrivals are characterized by non homogeneous Poisson process. It is further assumed that transmission time required by each packet at each node is dependent on the content of the buffer connected to it. The transient behavior of the network model is analyzed by deriving the system performance measures like mean number of packets in each buffer, mean delay in transmission, the throughput of the nodes, utilization of transmitters, etc,. The sensitivity analysis of the model reveals that the non homogeneous Poisson arrivals and dynamic bandwidth allocation strategy can reduce burstness in buffer and improve quality of service. A comparative study of communication network with non homogeneous Poisson arrivals and Poisson arrivals is also given.
\end{abstract}

\section{Keywords}

Communication Networks, Dynamic Bandwidth Allocation, non homogeneous Poisson Process.

\section{INTRODUCTION}

One of the important consideration in communication network models is transporting data/voice effectively with a guaranteed quality of service for accurate performance evaluation of communication network. Several network models have been developed with a wide variety of assumptions. These communication network models are much useful in developing and analyzing the systems at places like satellite communication, telecommunications, broad band integrated services, etc with accurate prediction of the performance measures (Huang et al (2010)).

Depending on the type of architecture used, the communication networks can be divided into three categories namely message switching, circuit switching and packet switching. It is generally known that the packet switching gives better utilization over message switching and circuit switching (Srinivasa Rao et al (2006)). In packet switching, the messages are divided into small packets of random length. Each packet will have an independent header in routing. The delay in packet switching can be reduced by utilizing statistical multiplexing. For efficient utilization of the resources the integration of statistical techniques with communication systems is needed. (Srinivas Rao K., et al (2000))

One of the most important aspect in developing communication networks is regarding the utilization of congestion control strategies. Usually bit dropping is employed for congestion control. The idea of bit dropping is to discard certain portion of the traffic such as least significant bit in order to reduce the transmission time while maintaining satisfactory quality of service (Kin K Leung (2002)). To improve the quality of service in transmission, several authors have studied the communication networks utilizing tandem queuing analogy (Kleinrock 1976, Yakuo Hayashda (1993), K. Sriram(1993)).

In all these papers they assumed that the constituent processes of the communication network namely, arrival and transmission processes are independent. But in some communication systems like store-and-forward communication systems the standard type of independent assumption is realistically inappropriate. Since the massages, generally preserve the length as they transfers the network, the inter arrival and service sequences at queue, interval to the system are time dependent as they formulate a queuing process at each node of the network through which the packet are routed. These dependences can have a significant influence on the system performance (Srinivas Rao K., et al(2001)).

Recently, some work has been reported in the literature regarding communication networks with dynamic bandwidth allocation/load dependent transmission for improving quality of service by utilizing ideal bandwidth (Suresh Varma $P$ et al(2007)., Padmavathi G et al (2009)., Nageswara Rao K et al (2010)). They considered that the arrival of messages for transmission are homogeneous. But in many practical situations arising places like satellite communication, wireless communication, telecommunication, computer communication, internet, WAN, the arrival of messages are to be considered as time dependent, in order to have accurate prediction of the performance measures of the system. In addition to this a number of measurements studies by Dinda (2006) have revealed 
that the traffic generated by many real world application exhibit a high degree of burstness (time varying arrival rates) and posses correlation in the number of message arrivals(Yulei W $\mathrm{N}$ et $\operatorname{al}(2009))$.

Therefore the traditional models with simplified assumption regarding message arrivals modeled by Poisson process cannot capture the bursty nature of message arrival process (Javadi et al 2007). Hence, it is needed to develop communication Network models with time dependent arrival rates. This type of time dependent arrival packets can be characterized by non homogeneous Poisson process.

Very little work has been reported in literature regarding communication network with non homogeneous Poisson arrivals. Hence, in this paper we develop and analyze a three node communication network model with non homogeneous Poisson arrivals having dynamic bandwidth allocation. In dynamic bandwidth allocation, the transmission rate of each packet is adjusted depending on the content of the buffer connected to the transmitter.

Using the difference-differential equations the probability generating function of the number of packets in each buffer is derived. The transient behavior of the communication network is analyzed by deriving the system performance measures like the mean content of the buffers, mean delay in transmission, throughput of nodes, utilization of transmitters, etc., explicitly. The sensitivity of the model with respect to the parameters is also carried. A comparative study of the communication network with non homogeneous Poisson arrivals and Poisson arrivals is also presented. This model includes some of the earlier models as particular cases for specific values of the parameters.

\section{THREE NODE COMMUNICATION NETWORK MODEL WITH DBA AND NHP ARRIVALS}

In this section, a communication network model having three nodes in tandem is studied. The arrivals to the buffer connected at node one are assumed to follow a non-homogeneous Poisson process with mean arrival rate as a function of time $t$. It is of the form $\lambda(t)=\lambda+\alpha t$. The transmission process from node one to node two follows a Poisson process with parameter $\mu_{1}$. After getting transmitted from node one the packets are forwarded to the second buffer for transmission. After getting transmitted from second node it is forwarded to the third buffer for transmission. The transmission processes of node two and three also follow Poisson processes with parameters $\mu_{2}$ and $\mu_{3}$ respectively. The transmission rate of each packet is adjusted just before transmission depending on the content of the buffer connected to the transmitter. The packets are transmitted through the transmitters by the first in first out discipline. The schematic diagram representing the communication network is shown in Figure 1

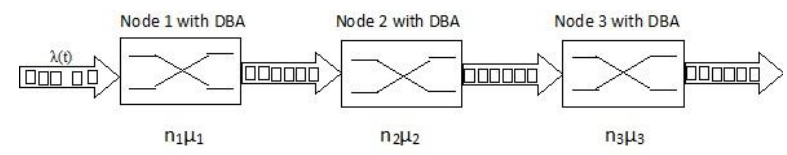

Figure 1 : Schematic diagram of the CNM model with 3 stage arrival.

Let $P_{n_{1}, n_{2}, n_{3}}(t)$ denote the probability that there are $n_{1}$ packets in the first buffer and $n_{2}$ packets in the second buffer and $\mathrm{n}_{3}$ packets in the third buffer at time $\mathrm{t}$

Then difference-differential equations of the network are

$$
\begin{aligned}
\frac{\partial P_{n_{1}, n_{2}, n_{3}}(t)}{\partial t}= & -\left(\lambda(t)+n_{1} \mu_{1}+n_{2} \mu_{2}+n_{3} \mu_{3}\right) P_{n_{1}, n_{2}, n_{3}}(t)+\lambda(t) P_{n_{1}-1, n_{2}, n_{3}}(t)+\left(n_{1}+1\right) \mu_{1} P_{n_{1}+1, n_{2}-1, n_{3}}(t) \\
& +\left(n_{2}+1\right) \mu_{2} P_{n_{1}, n_{2}+1, n_{3}-1}(t)+\left(n_{3}+1\right) \mu_{3} P_{n_{1}, n_{2}, n_{3}+1}(t), n_{1}, n_{2}, n_{3}>0 \\
\frac{\partial P_{0, n_{2}, n_{3}}(t)}{\partial t}= & -\left(\lambda(t)+n_{2} \mu_{2}+n_{3} \mu_{3}\right) P_{0, n_{2}, n_{3}}(t)+\mu_{1} P_{1, n_{2}-1, n_{3}}(t)+\left(n_{2}+1\right) \mu_{2} P_{0, n_{2}+1, n_{3}-1}(t) \\
& +\left(n_{3}+1\right) \mu_{3} P_{0, n_{2}, n_{3}+1}(t), n_{2}, n_{3}>0 \\
\frac{\partial P_{n_{1}, 0, n_{3}}(t)}{\partial t}= & -\left(\lambda(t)+n_{1} \mu_{1}+n_{3} \mu_{3}\right) P_{n_{1}, 0, n_{3}}(t)+\lambda(t) P_{n_{1}-1,0, n_{3}}(t)+\mu_{2} P_{n_{1}, n_{3}-1}(t)+\left(n_{3}+1\right) \mu_{3} P_{n_{1}, 0, n_{3}+1}(t) \\
\frac{\partial P_{n_{1}, n_{2}, 0}(t)}{\partial t}=- & -\left(\lambda(t)+n_{1} \mu_{1}+n_{2} \mu_{2}\right) P_{n_{1} n_{2}, 0}(t)+\lambda(t) P_{n_{1}-1, n_{2}, 0}(t)+\left(n_{1}+1\right) \mu_{1} P_{n_{1}+1, n_{2}-1,0}(t)+\mu_{3} P_{n_{1}, n_{2}, 1}(t) \\
\frac{\partial P_{0,0, n_{3}}, n_{2}>0}{\partial t}= & -\left(\lambda(t)+n_{3} \mu_{3}\right) P_{0,0, n_{3}}(t)+\mu_{2} P_{0,1, n_{3}-1}(t)+\left(n_{3}+1\right) \mu_{3} P_{0,0, n_{3}+1}(t), n_{3}>0 \\
\frac{\partial P_{n_{1}, 0,0}(t)}{\partial t}= & -\left(\lambda(t)+n_{1} \mu_{1}\right) P_{n_{1}, 0,0}(t)+\lambda(t) P_{n_{1}-1,0,0}(t)+\mu_{3} P_{n_{1}, 0,1}(t), n_{1}>0 \\
\frac{\partial P_{0, n_{2}, 0}(t)}{\partial t}= & -\left(\lambda(t)+n_{2} \mu_{2}\right) P_{0, n_{2}, 0}(t)+\mu_{1} P_{1, n_{2}-1,0}(t)+\mu_{3} P_{0, n_{2}, 1}(t), n_{2}>0 \\
\frac{\partial P_{0,0,0}(t)}{\partial t}= & -\lambda(t) P_{0,0,0}(t)+\mu_{3} P_{0,0,1}(t)
\end{aligned}
$$

Let $P\left(s_{1}, s_{2}, s_{3} ; t\right)=\sum_{n_{1}=0}^{\infty} \sum_{n_{2}=0}^{\infty} \sum_{n_{3}=0}^{\infty} P_{n_{1}, n_{2}, n_{3}}(t) s_{1}^{n_{1}} s_{2}^{n_{2}} s_{3}^{n_{3}} \quad$ be the joint probability generating function of $P_{n_{1}, n_{2}, n_{3}}(t)$.

Multiplying the equation (2.1) with $s_{1}{ }^{n_{1}}, s_{2}{ }^{n_{2}}, s_{3}{ }^{{ }_{3}}$ and summing over all $\mathrm{n}_{1}, \mathrm{n}_{2}$, and $\mathrm{n}_{3}$ we get

$$
\begin{aligned}
\frac{d P\left(s_{1}, s_{2}, s_{3} ; t\right)}{d t}= & -\lambda(t) P\left(s_{1}, s_{2}, s_{3} ; t\right)+\lambda(t) s_{1} P\left(s_{1}, s_{2}, s_{3} ; t\right)-\mu_{1} s_{1} \frac{\partial P\left(s_{1}, s_{2}, s_{3} ; t\right)}{\partial s_{1}}+\mu_{1} s_{2} \frac{\partial P\left(s_{1}, s_{2}, s_{3} ; t\right)}{\partial s_{1}} \\
& -\mu_{2} s_{2} \frac{\partial P\left(s_{1}, s_{2}, s_{3} ; t\right)}{\partial s_{2}}+\mu_{2} s_{3} \frac{\partial P\left(s_{1}, s_{2}, s_{3} ; t\right)}{\partial s_{2}}-\mu_{3} s_{3} \frac{\partial P\left(s_{1}, s_{2}, s_{3} ; t\right)}{\partial s_{3}}+\mu_{3} \frac{\partial P\left(s_{1}, s_{2}, s_{3} ; t\right)}{\partial s_{3}}
\end{aligned}
$$

After simplifying, we get

$$
\begin{aligned}
\frac{\partial P\left(s_{1}, s_{2}, s_{3} ; t\right)}{\partial t}= & \mu_{1}\left(s_{2}-s_{1}\right) \frac{\partial P\left(s_{1}, s_{2}, s_{3} ; t\right)}{\partial s_{1}}+\mu_{2}\left(s_{3}-s_{2}\right) \frac{\partial P\left(s_{1}, s_{2}, s_{3} ; t\right)}{\partial s_{2}}+\mu_{3}\left(1-s_{3}\right) \frac{\partial P\left(s_{1}, s_{2}, s_{3} ; t\right)}{\partial s_{3}} \\
& +\lambda(t) P\left(s_{1}, s_{2}, s_{3} ; t\right)\left(s_{1}-1\right)
\end{aligned}
$$

Solving the equation (2.4) by Lagrangian's method, the auxiliary equations are

$$
\frac{d t}{1}=\frac{d s_{1}}{\mu_{1}\left(s_{1}-s_{2}\right)}=\frac{d s_{2}}{\mu_{2}\left(s_{2}-s_{3}\right)}=\frac{d s_{3}}{\mu_{2}\left(s_{3}-1\right)}=\frac{d P}{\lambda(t) P\left(s_{1}, s_{2}, s_{3} ; t\right)\left(s_{1}-1\right)}
$$


To solve the equations in (2.5) the functional form of $\lambda(t)$ is required. Let the mean arrival rate of packets is $\lambda(t)=\lambda+\alpha t$, where $\lambda>0, \alpha>0$ are constants.

Solving the first and fourth terms in equation (2.5), we get

$$
a=\left(s_{3}-1\right) e^{-\mu_{3} t}
$$

Solving the first and third terms in equation (2.5), we get

$$
b=\left(s_{2}-1\right) e^{-\mu_{2} t}+\frac{\mu_{2}}{\mu_{3}-\mu_{2}}\left(s_{3}-1\right) e^{-\mu_{2} t}
$$

Solving the first and second terms in equation (2.5), we get

$$
c=\left(s_{1}-1\right) e^{-\mu_{1} t}+\frac{\mu_{1}}{\mu_{2}-\mu_{1}}\left(s_{2}-1\right) e^{-\mu_{1} t}+\frac{\mu_{1} \mu_{2}}{\left(\mu_{3}-\mu_{1}\right)\left(\mu_{2}-\mu_{1}\right)}\left(s_{3}-1\right) e^{-\mu_{1} t}
$$

Solving the first and fifth terms in equation (2.5), we get

$$
\begin{array}{r}
d=P\left(s_{1}, s_{2}, s_{3} ; t\right) \exp \left\{-\left[\frac{\left(s_{1}-1\right)}{\mu_{1}}\left(\lambda+\alpha t-\frac{\alpha}{\mu_{1}}\right)+\frac{\left(s_{2}-1\right)}{\mu_{2}}\left(\lambda+\alpha t-\frac{\alpha\left(\mu_{1}+\mu_{2}\right)}{\mu_{1} \mu_{2}}\right)\right.\right. \\
\left.\left.+\frac{\left(s_{3}-1\right)}{\mu_{3}}\left(\lambda+\alpha t-\frac{\alpha\left(\mu_{1} \mu_{2}+\mu_{2} \mu_{3}+\mu_{1} \mu_{3}\right)}{\mu_{1} \mu_{2} \mu_{3}}\right)\right]\right\}
\end{array}
$$

where, a, b, c and d ar arbitrary constants. Using the initiale conditions $P_{000}(0)=1, \quad P_{000}(t)=0 \quad \forall t>0$

The general solution of (2.5) gives the probability generating function of the number of packets in the first, second and third buffers at time $\mathrm{t}$, as $\mathrm{P}\left(\mathrm{s}_{1}, \mathrm{~s}_{2}, \mathrm{~s}_{3,} ; \mathrm{t}\right)$.

Therefore

$$
\begin{array}{r}
P\left(s_{1}, s_{2}, s_{3} ; t\right)=\exp \left\{\frac{\left(s_{1}-1\right)}{\mu_{1}}\left(1-e^{-\mu_{1} t}\right)\left(\lambda-\frac{\alpha}{\mu_{1}}\right)+\frac{\left(s_{1}-1\right) \alpha t}{\mu_{1}}+\frac{\left(s_{2}-1\right)}{\mu_{2}}\left(1-e^{-\mu_{2} t}\right)\left(\lambda-\alpha\left(\frac{1}{\mu_{2}}+\frac{1}{\mu_{1}}\right)\right)\right. \\
+\frac{\left(s_{2}-1\right) \alpha t}{\mu_{2}}+\frac{\left(s_{2}-1\right)}{\mu_{2}-\mu_{1}}\left(e^{-\mu_{2} t}-e^{-\mu_{1} t}\right)\left(\lambda-\frac{\alpha}{\mu_{1}}\right)+\frac{\left(s_{3}-1\right)}{\mu_{3}}\left(1-e^{-\mu_{3} t}\right)\left(\lambda-\alpha\left(\frac{1}{\mu_{3}}+\frac{1}{\mu_{2}}+\frac{1}{\mu_{1}}\right)\right) \\
+\frac{\left(s_{3}-1\right) \alpha t}{\mu_{3}}+\frac{\left(s_{3}-1\right)}{\mu_{3}-\mu_{2}}\left(e^{-\mu_{3} t}-e^{-\mu_{2} t}\right)\left(\lambda-\alpha\left(\frac{1}{\mu_{2}}+\frac{1}{\mu_{1}}\right)\right) \\
\left.+\left(s_{3}-1\right) \mu_{2}\left(\frac{e^{-\mu_{3} t}}{\left(\mu_{2}-\mu_{3}\right)\left(\mu_{3}-\mu_{1}\right)}+\frac{e^{-\mu_{2} t}}{\left(\mu_{3}-\mu_{2}\right)\left(\mu_{2}-\mu_{1}\right)}+\frac{e^{-\mu_{1} t}}{\left(\mu_{1}-\mu_{3}\right)\left(\mu_{2}-\mu_{1}\right)}\right)\left(\lambda-\frac{\alpha}{\mu_{1}}\right)\right\}
\end{array}
$$$$
\text { for } \lambda<\min \left\{\mu_{1}, \mu_{2}, \mu_{3}\right\}
$$

\section{PERFORMANCE MEASURE OF THE NETWORK:}

In this section, we derive and analyze the performance measures of the communication network under transient conditions. Expanding $\mathrm{P}\left(\mathrm{s}_{1}, \mathrm{~s}_{2}, \mathrm{~s}_{3} ; \mathrm{t}\right)$ given in equation (2.7) and collecting the constant terms, we get the probability that the network is empty as

$$
\begin{aligned}
P_{000}(t)= & \exp \left\{-\left[\frac{1}{\mu_{1}}\left(1-e^{-\mu_{1} t}\right)\left(\lambda-\frac{\alpha}{\mu_{1}}\right)+\frac{\alpha t}{\mu_{1}}+\frac{1}{\mu_{2}}\left(1-e^{-\mu_{2} t}\right)\left(\lambda-\alpha\left(\frac{1}{\mu_{2}}+\frac{1}{\mu_{1}}\right)\right)+\frac{\alpha t}{\mu_{2}}\right.\right. \\
& +\frac{1}{\mu_{2}-\mu_{1}}\left(e^{-\mu_{2} t}-e^{-\mu_{1} t}\right)\left(\lambda-\frac{\alpha}{\mu_{1}}\right)+\frac{1}{\mu_{3}}\left(1-e^{-\mu_{3} t}\right)\left(\lambda-\alpha\left(\frac{1}{\mu_{3}}+\frac{1}{\mu_{2}}+\frac{1}{\mu_{1}}\right)\right)+\frac{\alpha t}{\mu_{3}} \\
& +\frac{1}{\left(\mu_{3}-\mu_{2}\right)}\left(e^{-\mu_{3} t}-e^{-\mu_{2} t}\right)\left(\lambda-\alpha\left(\frac{1}{\mu_{2}}+\frac{1}{\mu_{1}}\right)\right) \\
& \left.\left.+\mu_{2}\left(\frac{e^{-\mu_{3} t}}{\left(\mu_{2}-\mu_{3}\right)\left(\mu_{3}-\mu_{1}\right)}+\frac{e^{-\mu_{2} t}}{\left(\mu_{3}-\mu_{2}\right)\left(\mu_{2}-\mu_{1}\right)}+\frac{e^{-\mu_{1} t}}{\left(\mu_{1}-\mu_{3}\right)\left(\mu_{2}-\mu_{1}\right)}\right)\left(\lambda-\frac{\alpha}{\mu_{1}}\right)\right]\right\}
\end{aligned}
$$

Taking $s_{2}=1, s_{3}=1$ in equation (2.7), we get the probability generating function of the first buffer size as

$P\left(s_{1}, t\right)=\exp \left\{\frac{\left(s_{1}-1\right)}{\mu_{1}}\left(\lambda-\frac{\alpha}{\mu_{1}}\right)\left(1-e^{-\mu_{1} t}\right)+\frac{\left(s_{1}-1\right)}{\mu_{1}} \alpha t\right\}$ for $\lambda<\mu_{1}$.

Expanding $\mathrm{P}\left(\mathrm{s}_{1}, \mathrm{t}\right)$ and collecting the constant terms, we get the probability that the first buffer is empty as

$$
P_{0 . .}(t)=\exp \left\{-\left[\frac{1}{\mu_{1}}\left(\lambda-\frac{\alpha}{\mu_{1}}\right)\left(1-e^{-\mu_{1} t}\right)+\frac{1}{\mu_{1}} \alpha t\right]\right\}
$$

Similarly taking $s_{1}=1, s_{2}=1$ in equation (2.7), we get the probability generating function of the second buffer size as

$$
\begin{gathered}
P\left(s_{2}, t\right)=\exp \left\{\left[\frac{\left(s_{2}-1\right)}{\mu_{2}}\left(1-e^{-\mu_{2}}\right)\left(\lambda-\alpha\left(\frac{1}{\mu_{2}}+\frac{1}{\mu_{1}}\right)\right)+\frac{\left(s_{2}-1\right)}{\mu_{2}} \alpha t+\frac{\left(s_{2}-1\right)}{\mu_{2}-\mu_{1}}\left(e^{-\mu_{2} t}-e^{-\mu_{1} t}\right)\left(\lambda-\frac{\alpha}{\mu_{1}}\right)\right]\right\} \\
\text { for } \lambda<\min \left\{\mu_{1}, \mu_{2}\right\}
\end{gathered}
$$

Expanding $\mathrm{P}\left(\mathrm{s}_{2}, \mathrm{t}\right)$ and collecting the constant terms, we get the probability that the second buffer is empty as

$$
P_{0.0}(t)=\exp \left\{-\left[\frac{1}{\mu_{2}}\left(1-e^{-\mu_{2}}\right)\left(\lambda-\alpha\left(\frac{1}{\mu_{2}}+\frac{1}{\mu_{1}}\right)\right)+\frac{1}{\mu_{2}} \alpha t+\frac{1}{\mu_{2}-\mu_{1}}\left(e^{-\mu_{2} t}-e^{-\mu_{1} t}\right)\left(\lambda-\frac{\alpha}{\mu_{1}}\right)\right]\right\}
$$

Similarly taking $s_{1}=1, s_{2}=1$ in equation (2.7), we get the probability generating function of the third buffer size as

$$
\begin{array}{r}
P\left(s_{3}, t\right)=\exp \left\{\frac{\left(s_{3}-1\right)}{\mu_{3}}\left(1-e^{-\mu_{3}}\right)\left(\lambda-\alpha\left(\frac{1}{\mu_{3}}+\frac{1}{\mu_{2}}+\frac{1}{\mu_{1}}\right)\right)+\frac{\left(s_{3}-1\right) \alpha t}{\mu_{3}}+\frac{\left(s_{3}-1\right)}{\mu_{3}-\mu_{2}}\left(e^{-\mu_{3}}-e^{-\mu_{2}}\right)\left(\lambda-\alpha\left(\frac{1}{\mu_{2}}+\frac{1}{\mu_{1}}\right)\right)\right. \\
\left.+\left(s_{3}-1\right) \mu_{2}\left(\frac{e^{-\mu_{3}}}{\left(\mu_{2}-\mu_{3}\right)\left(\mu_{3}-\mu_{1}\right)}+\frac{e^{-\mu_{2}}}{\left(\mu_{3}-\mu_{2}\right)\left(\mu_{2}-\mu_{1}\right)}+\frac{e^{-\mu_{t}}}{\left(\mu_{1}-\mu_{3}\right)\left(\mu_{2}-\mu_{1}\right)}\right)\left(\lambda-\frac{\alpha}{\mu_{1}}\right)\right\}
\end{array}
$$

for $\lambda<\min \left\{\mu_{1}, \mu_{2}, \mu_{3}\right\}$

Expanding $\mathrm{P}\left(\mathrm{s}_{3}, \mathrm{t}\right)$ and collecting the constant terms, we get the probability that the third buffer is empty as

$$
\begin{array}{r}
P_{.0}(t)=\exp \left\{-\left[\frac{1}{\mu_{3}}\left(1-e^{-\mu_{s}}\right)\left(\lambda-\alpha\left(\frac{1}{\mu_{3}}+\frac{1}{\mu_{2}}+\frac{1}{\mu_{1}}\right)\right)+\frac{\alpha t}{\mu_{3}}+\frac{1}{\mu_{3}-\mu_{2}}\left(e^{-\mu_{t} t}-e^{-\mu_{2}}\right)\left(\lambda-\alpha\left(\frac{1}{\mu_{2}}+\frac{1}{\mu_{1}}\right)\right)\right.\right. \\
\left.\left.+\mu_{2}\left(\frac{e^{-\mu_{j}}}{\left(\mu_{2}-\mu_{3}\right)\left(\mu_{3}-\mu_{1}\right)}+\frac{e^{-\mu_{t} t}}{\left(\mu_{3}-\mu_{2}\right)\left(\mu_{2}-\mu_{1}\right)}+\frac{e^{-\mu_{t}}}{\left(\mu_{1}-\mu_{3}\right)\left(\mu_{2}-\mu_{1}\right)}\right)\left(\lambda-\frac{\alpha}{\mu_{1}}\right)\right]\right\}
\end{array}
$$

The mean number of packets in the first buffer is 
$L_{1}(t)=\frac{1}{\mu_{1}}\left(\lambda-\frac{\alpha}{\mu_{1}}\right)\left(1-e^{-\mu_{1} t}\right)+\frac{1}{\mu_{1}} \alpha t$

The utilization of the first transmitter is

$U_{1}(t)=1-\exp \left\{-\left[\frac{1}{\mu_{1}}\left(\lambda-\frac{\alpha}{\mu_{1}}\right)\left(1-e^{-\mu_{1} t}\right)+\frac{1}{\mu_{1}} \alpha t\right]\right\}$

The mean number of the packets in second buffer is

$L_{2}(t)=\frac{1}{\mu_{2}}\left(1-e^{-\mu_{2} t}\right)\left(\lambda-\alpha\left(\frac{1}{\mu_{2}}+\frac{1}{\mu_{1}}\right)\right)+\frac{1}{\mu_{2}} \alpha t+\frac{1}{\mu_{2}-\mu_{1}}\left(e^{-\mu_{2} t}-e^{-\mu_{1} t}\right)\left(\lambda-\frac{\alpha}{\mu_{1}}\right)$

The utilization of the second transmitter is

$U_{2}(t)=1-\exp \left\{-\left[\frac{1}{\mu_{2}}\left(1-e^{-\mu_{2} t}\right)\left(\lambda-\alpha\left(\frac{1}{\mu_{2}}+\frac{1}{\mu_{1}}\right)\right)+\frac{1}{\mu_{2}} \alpha t+\frac{1}{\mu_{2}-\mu_{1}}\left(e^{-\mu_{2} t}-e^{-\mu_{1} t}\right)\left(\lambda-\frac{\alpha}{\mu_{1}}\right)\right]\right\}$

The mean number of the packets in third buffer is

$$
\begin{aligned}
L_{3}(t)= & {\left[\frac{1}{\mu_{3}}\left(1-e^{-\mu t_{t}}\right)\left(\lambda-\alpha\left(\frac{1}{\mu_{3}}+\frac{1}{\mu_{2}}+\frac{1}{\mu_{1}}\right)\right)+\frac{\alpha t}{\mu_{3}}+\frac{1}{\mu_{3}-\mu_{2}}\left(e^{-\mu_{3} t}-e^{-\mu_{2} t}\right)\left(\lambda-\alpha\left(\frac{1}{\mu_{2}}+\frac{1}{\mu_{1}}\right)\right)\right.} \\
& \left.+\mu_{2}\left(\frac{e^{-\mu_{3} t}}{\left(\mu_{2}-\mu_{3}\right)\left(\mu_{3}-\mu_{1}\right)}+\frac{e^{-\mu_{2} t}}{\left(\mu_{3}-\mu_{2}\right)\left(\mu_{2}-\mu_{1}\right)}+\frac{e^{-\mu_{t} t}}{\left(\mu_{1}-\mu_{3}\right)\left(\mu_{2}-\mu_{1}\right)}\right)\left(\lambda-\frac{\alpha}{\mu_{1}}\right)\right]
\end{aligned}
$$

The utilization of the third transmitter is

$$
\begin{array}{r}
U_{3}(t)=1-\exp \left\{-\left[\frac{1}{\mu_{3}}\left(1-e^{-\mu_{1}}\right)\left(\lambda-\alpha\left(\frac{1}{\mu_{3}}+\frac{1}{\mu_{2}}+\frac{1}{\mu_{1}}\right)\right)+\frac{\alpha t}{\mu_{3}}+\frac{1}{\mu_{3}-\mu_{2}}\left(e^{-\mu_{3}}-e^{-\mu_{2}}\right)\left(\lambda-\alpha\left(\frac{1}{\mu_{2}}+\frac{1}{\mu_{1}}\right)\right)\right.\right. \\
\left.\left.+\mu_{2}\left(\frac{e^{-\mu_{3} t}}{\left(\mu_{2}-\mu_{3}\right)\left(\mu_{3}-\mu_{1}\right)}+\frac{e^{-\mu_{2} t}}{\left(\mu_{3}-\mu_{2}\right)\left(\mu_{2}-\mu_{1}\right)}+\frac{e^{-\mu_{1}}}{\left(\mu_{1}-\mu_{3}\right)\left(\mu_{2}-\mu_{1}\right)}\right)\left(\lambda-\frac{\alpha}{\mu_{1}}\right)\right]\right\}
\end{array}
$$

The variance of the number of packets in the first buffer is

$\mathrm{V}_{1}(\mathrm{t})=\frac{1}{\mu_{1}}\left(\lambda-\frac{\alpha}{\mu_{1}}\right)\left(1-e^{-\mu_{1} t}\right)+\frac{1}{\mu_{1}} \alpha t$

The variance of the number of packets in the second buffer is

$$
\mathrm{V}_{2}(\mathrm{t})=\frac{1}{\mu_{2}}\left(1-e^{-\mu_{2} t}\right)\left(\lambda-\alpha\left(\frac{1}{\mu_{2}}+\frac{1}{\mu_{1}}\right)\right)+\frac{1}{\mu_{2}} \alpha t+\frac{1}{\mu_{2}-\mu_{1}}\left(e^{-\mu_{2} t}-e^{-\mu_{1} t}\right)\left(\lambda-\frac{\alpha}{\mu_{1}}\right)
$$

The variance of the number of packets in the third buffer is

$$
\begin{array}{r}
\mathrm{V}_{3}(\mathrm{t})=\frac{1}{\mu_{3}}\left(1-e^{-\mu_{3} t}\right)\left(\lambda-\alpha\left(\frac{1}{\mu_{3}}+\frac{1}{\mu_{2}}+\frac{1}{\mu_{1}}\right)\right)+\frac{\alpha t}{\mu_{3}}+\frac{1}{\mu_{3}-\mu_{2}}\left(e^{-\mu_{3} t}-e^{-\mu_{2} t}\right)\left(\lambda-\alpha\left(\frac{1}{\mu_{2}}+\frac{1}{\mu_{1}}\right)\right) \\
+\mu_{2}\left(\frac{e^{-\mu_{3} t}}{\left(\mu_{2}-\mu_{3}\right)\left(\mu_{3}-\mu_{1}\right)}+\frac{e^{-\mu_{2} t}}{\left(\mu_{3}-\mu_{2}\right)\left(\mu_{2}-\mu_{1}\right)}+\frac{e^{-\mu_{1} t}}{\left(\mu_{1}-\mu_{3}\right)\left(\mu_{2}-\mu_{1}\right)}\right)\left(\lambda-\frac{\alpha}{\mu_{1}}\right)
\end{array}
$$

The throughput of the first transmitter is

$$
\mu_{1}\left(1-P_{0 . .}(t)\right)=\mu_{1}\left[1+\exp \left\{\frac{1}{\mu_{1}}\left(\lambda-\frac{\alpha}{\mu_{1}}\right)\left(1-e^{-\mu_{1} t}\right)+\frac{1}{\mu_{1}} \alpha t\right\}\right]
$$

The mean delay in the first buffer is

$$
W_{1}(t)=\frac{\frac{1}{\mu_{1}}\left(\lambda-\frac{\alpha}{\mu_{1}}\right)\left(1-e^{-\mu_{1} t}\right)+\frac{1}{\mu_{1}} \alpha t}{\mu_{1}\left[1+\exp \left\{\frac{1}{\mu_{1}}\left(\lambda-\frac{\alpha}{\mu_{1}}\right)\left(1-e^{-\mu_{1} t}\right)+\frac{1}{\mu_{1}} \alpha t\right\}\right]}
$$

The throughput of the second transmitter is

$\mu_{2}\left(1-P_{.0}(t)\right)=\mu_{2}\left[1+\exp \left\{\left[\frac{1}{\mu_{2}}\left(1-e^{-\mu_{2} t}\right)\left(\lambda-\alpha\left(\frac{1}{\mu_{2}}+\frac{1}{\mu_{1}}\right)\right)+\frac{1}{\mu_{2}} \alpha t+\frac{1}{\mu_{2}-\mu_{1}}\left(e^{-\mu_{2} t}-e^{-\mu_{1} t}\right)\left(\lambda-\frac{\alpha}{\mu_{1}}\right)\right]\right\}\right]$

The mean delay in the second buffer is

$$
W_{2}(t)=\frac{\frac{1}{\mu_{2}}\left(1-e^{-\mu_{2}}\right)\left(\lambda-\alpha\left(\frac{1}{\mu_{2}}+\frac{1}{\mu_{1}}\right)\right)+\frac{1}{\mu_{2}} \alpha t+\frac{1}{\mu_{2}-\mu_{1}}\left(e^{-\mu_{2} t}-e^{-\mu_{1}}\right)\left(\lambda-\frac{\alpha}{\mu_{1}}\right)}{\mu_{2}\left[1+\exp \left[\frac{1}{\mu_{2}}\left(1-e^{-\mu_{2}}\right)\left(\lambda-\alpha\left(\frac{1}{\mu_{2}}+\frac{1}{\mu_{1}}\right)+\frac{1}{\mu_{2}} \alpha t+\frac{1}{\mu_{2}-\mu_{1}}\left(e^{-\mu_{2} t}-e^{-\mu_{1} t}\right)\left(\lambda-\frac{\alpha}{\mu_{1}}\right)\right]\right]\right.}
$$

The throughput of the third transmitter is

$$
\begin{array}{r}
\mu_{3}\left(1-P_{.0}(t)\right)=\mu_{3}\left[1+\exp \left[\frac{1}{\mu_{3}}\left(1-e^{-\mu_{3} t}\right)\left(\lambda-\alpha\left(\frac{1}{\mu_{3}}+\frac{1}{\mu_{2}}+\frac{1}{\mu_{1}}\right)\right)+\frac{\alpha t}{\mu_{3}}+\frac{1}{\mu_{3}-\mu_{2}}\left(e^{-\mu_{3} t}-e^{-\mu_{2} t}\right)\left(\lambda-\alpha\left(\frac{1}{\mu_{2}}+\frac{1}{\mu_{1}}\right)\right)\right.\right. \\
\left.\left.+\mu_{2}\left(\frac{e^{-\mu_{3} t}}{\left(\mu_{2}-\mu_{3}\right)\left(\mu_{3}-\mu_{1}\right)}+\frac{e^{-\mu_{2} t}}{\left(\mu_{3}-\mu_{2}\right)\left(\mu_{2}-\mu_{1}\right)}+\frac{e^{-\mu_{t} t}}{\left(\mu_{1}-\mu_{3}\right)\left(\mu_{2}-\mu_{1}\right)}\right)\left(\lambda-\frac{\alpha}{\mu_{1}}\right)\right]\right]
\end{array}
$$

The mean delay in the third buffer is

$$
\begin{array}{r}
\frac{1}{\mu_{3}}\left(1-e^{-\mu_{1}}\right)\left(\lambda-\alpha\left(\frac{1}{\mu_{3}}+\frac{1}{\mu_{2}}+\frac{1}{\mu_{1}}\right)\right)+\frac{\alpha t}{\mu_{3}}+\frac{1}{\mu_{3}-\mu_{2}}\left(e^{-\mu_{t} t}-e^{-\mu_{1} t}\right)\left(\lambda-\alpha\left(\frac{1}{\mu_{2}}+\frac{1}{\mu_{1}}\right)\right) \\
W_{3}(t)=\frac{+\mu_{2}\left(\frac{e^{-\mu_{3}}}{\left(\mu_{2}-\mu_{3}\right)\left(\mu_{3}-\mu_{1}\right)}+\frac{e^{-\mu_{3}}}{\left(\mu_{3}-\mu_{2}\right)\left(\mu_{2}-\mu_{1}\right)}+\frac{e^{-\mu_{t}}}{\left(\mu_{1}-\mu_{3}\right)\left(\mu_{2}-\mu_{1}\right)}\right)\left(\lambda-\frac{\alpha}{\mu_{1}}\right)}{\mu_{3}\left[1+\exp \left[\frac{1}{\mu_{3}}\left(1-e^{\left.-\mu_{3}\right)}\right)\left(\lambda-\alpha\left(\frac{1}{\mu_{3}}+\frac{1}{\mu_{2}}+\frac{1}{\mu_{1}}\right)\right)+\frac{\alpha t}{\mu_{3}}+\frac{1}{\mu_{3}-\mu_{2}}\left(e^{-\mu_{3}}-e^{-\mu_{3}}\right)\left(\lambda-\alpha\left(\frac{1}{\mu_{2}}+\frac{1}{\mu_{1}}\right)\right)\right.\right.} \\
\left.\left.+\mu_{2}\left(\frac{e^{-\mu_{3}}}{\left(\mu_{2}-\mu_{3}\right)\left(\mu_{3}-\mu_{1}\right)}+\frac{e^{-\mu_{2}}}{\left(\mu_{3}-\mu_{2}\right)\left(\mu_{2}-\mu_{1}\right)}+\frac{e^{-\mu_{t}}}{\left(\mu_{1}-\mu_{3}\right)\left(\mu_{2}-\mu_{1}\right)}\right)\left(\lambda-\frac{\alpha}{\mu_{1}}\right)\right]\right]
\end{array}
$$

The mean number of packets in the entire network at time $\mathrm{t}$ is 


$$
\begin{aligned}
L(t)= & \frac{1}{\mu_{1}}\left(1-e^{-\mu_{1} t}\right)\left(\lambda-\frac{\alpha}{\mu_{1}}\right)+\frac{1}{\mu_{1}} \alpha t+\frac{1}{\mu_{2}}\left(1-e^{-\mu_{2} t}\right)\left(\lambda-\alpha\left(\frac{1}{\mu_{2}}+\frac{1}{\mu_{1}}\right)\right)+\frac{1}{\mu_{2}} \alpha t \\
& +\frac{1}{\mu_{2}-\mu_{1}}\left(e^{-\mu_{2} t}-e^{-\mu_{1} t}\right)\left(\lambda-\frac{\alpha}{\mu_{1}}\right)+\frac{1}{\mu_{3}}\left(1-e^{-\mu_{3} t}\right)\left(\lambda-\alpha\left(\frac{1}{\mu_{3}}+\frac{1}{\mu_{2}}+\frac{1}{\mu_{1}}\right)\right) \\
& +\frac{\alpha t}{\mu_{3}}+\frac{1}{\mu_{3}-\mu_{2}}\left(e^{-\mu_{3} t}-e^{-\mu_{2} t}\right)\left(\lambda-\alpha\left(\frac{1}{\mu_{2}}+\frac{1}{\mu_{1}}\right)\right) \\
& +\mu_{2}\left(\frac{e^{-\mu_{3} t}}{\left(\mu_{2}-\mu_{3}\right)\left(\mu_{3}-\mu_{1}\right)}+\frac{e^{-\mu_{2} t}}{\left(\mu_{3}-\mu_{2}\right)\left(\mu_{2}-\mu_{1}\right)}+\frac{e^{-\mu_{1} t}}{\left(\mu_{1}-\mu_{3}\right)\left(\mu_{2}-\mu_{1}\right)}\right)\left(\lambda-\frac{\alpha}{\mu_{1}}\right)
\end{aligned}
$$

The variability of the number of packets in the network is

$$
\begin{aligned}
\mathrm{V}(t) & =\frac{1}{\mu_{1}}\left(1-e^{-\mu_{1} t}\right)\left(\lambda-\frac{\alpha}{\mu_{1}}\right)+\frac{1}{\mu_{1}} \alpha t+\frac{1}{\mu_{2}}\left(1-e^{-\mu_{2} t}\right)\left(\lambda-\alpha\left(\frac{1}{\mu_{2}}+\frac{1}{\mu_{1}}\right)\right)+\frac{1}{\mu_{2}} \alpha t \\
& +\frac{1}{\mu_{2}-\mu_{1}}\left(e^{-\mu_{2} t}-e^{-\mu_{1} t}\right)\left(\lambda-\frac{\alpha}{\mu_{1}}\right)+\frac{1}{\mu_{3}}\left(1-e^{-\mu_{3} t}\right)\left(\lambda-\alpha\left(\frac{1}{\mu_{3}}+\frac{1}{\mu_{2}}+\frac{1}{\mu_{1}}\right)\right) \\
& +\frac{\alpha t}{\mu_{3}}+\frac{1}{\mu_{3}-\mu_{2}}\left(e^{-\mu_{3} t}-e^{-\mu_{2} t}\right)\left(\lambda-\alpha\left(\frac{1}{\mu_{2}}+\frac{1}{\mu_{1}}\right)\right) \\
& +\mu_{2}\left(\frac{e^{-\mu_{3} t}}{\left(\mu_{2}-\mu_{3}\right)\left(\mu_{3}-\mu_{1}\right)}+\frac{e^{-\mu_{2} t}}{\left(\mu_{3}-\mu_{2}\right)\left(\mu_{2}-\mu_{1}\right)}+\frac{e^{-\mu_{t} t}}{\left(\mu_{1}-\mu_{3}\right)\left(\mu_{2}-\mu_{1}\right)}\right)\left(\lambda-\frac{\alpha}{\mu_{1}}\right)
\end{aligned}
$$

\section{PERFORMANCE EVALUATION OF THE NETWORK}

In this section, the performance of the communication network is discussed through numerical illustration. Different values of the parameters are considered for bandwidth allocation and arrival of packets. After interacting with the technical staff at internet providing station, it is considered that the packet arrival parameter $(\lambda)$ varies from $2 \times 10^{4}$ packets/sec to $7 \times 10^{4}$ packets/sec, and $(\alpha)$ varies from 1 to 3 with an average packet size of 53 bytes. After transmitting from node 1, the forward transmission rate $\left(\mu_{1}\right)$ varies from $5 \times 10^{4}$ packets/sec to $9 \times 10^{4}$ packets/sec. The rate of transmission from node $2\left(\mu_{2}\right)$ varies from $15 \times 10^{4}$ packets/sec to $19 \times 10^{4}$ packets/sec. The rate of transmission from node $3\left(\mu_{3}\right)$ varies from $25 \times 10^{4}$ packets $/ \mathrm{sec}$ to $29 \times 10^{4}$ packets/sec. In all the nodes, the dynamic bandwidth allocation strategy is considered i.e., the transmission rate of each packet depends on the number of packets in the buffer connected to it at that instant.

Using equations (3.9), (3.11), (3.13), (3.17), (3.19), and (3.21) the utilization of the transmitters and throughput of three nodes are computed for different values of the parameters $t, \lambda, \alpha, \mu_{1}, \mu_{2}$, $\mu_{3}$ and are presented in table 1 . The relationship between parameters and utilization of transmitters and throughput of nodes are shown in Figure.1.

From table 1, it is observed that as the time (t) and arrival parameter $(\lambda)$ increases, the utilization of transmitters are increasing for fixed values of the other parameters. It is also observed that as the parameter $(\alpha)$ increases, the utilization of transmitters at all nodes are increasing for fixed values of the other parameters. As the transmission parameter $\left(\mu_{1}\right)$ increases, the utilization of the first node decreases and utilization of second and third nodes increase when the other parameters remain fixed. Similarly, as the transmission parameter $\left(\mu_{2}\right)$ increases the utilization of the first node is constant, the utilization of the second node decreases and utilization of the third node increases when other parameters remain fixed. Similarly, as the transmission parameter $\left(\mu_{3}\right)$ increases the utilization of the first and second nodes are constant and the utilization of the third node decreases when other parameters remain fixed.

It is observed as time value $t$ increases, the throughput of first, second and third nodes are increasing for fixed values of the other parameters. As the parameter $(\lambda)$ varies from $3 \times 10^{4}$ packets/sec to $7 \times 10^{4}$ packets/sec, the throughput of the first

\begin{tabular}{|c|c|c|c|c|c|c|c|c|c|c|c|}
\hline & $\lambda$ & $\alpha$ & $\mu_{1}^{s}$ & $\mu_{2}^{s}$ & $\mu_{3}^{s}$ & $U_{1}(t)$ & $U_{2}(t)$ & $U_{3}(t)$ & $\mathrm{hp}_{1}(\mathrm{t})$ & $\operatorname{Thp}_{2}(\mathrm{t})$ & $\mathrm{hp}_{3}(\mathrm{t})$ \\
\hline & 2 & 1 & 5 & 5 & 5 & 260 & 27054 & & & & \\
\hline & 2 & 1 & 5 & & & & 634 & 60796 & & 7451 & \\
\hline & & 1 & & & & & & & & & \\
\hline & & 1 & 5 & & & & & & & & \\
\hline & 2 & 1 & 5 & & & & 9330 & & & & \\
\hline & & 1 & 5 & & & & & & & & \\
\hline & 2 & 1 & 5 & 15 & & 43339 & 361663 & 234888 & 71670 & 42494 & 5.8722 \\
\hline & & 1 & 5 & & & & & & & & \\
\hline & 4 & 1 & 5 & & & & & & & & \\
\hline & 5 & 1 & 5 & & & & & & & & \\
\hline & 0 & 1 & 5 & J & & & & & & & \\
\hline .5 & 7 & 1 & 5 & 15 & 25 & & & & & & \\
\hline & 2 & -0.5 & 5 & & & & & & & & \\
\hline & 2 & 0 & 5 & 15 & & & & & & & \\
\hline & 2 & 0.5 & 5 & & & & & & & & \\
\hline & 2 & 1 & 5 & 15 & 25 & & & & & & \\
\hline 0.5 & 2 & 1.5 & 5 & 15 & 25 & & 3027 & 890 & & & \\
\hline & 2 & 1 & 5 & 15 & & & & & & & \\
\hline & 2 & 1 & 6 & 15 & 25 & & & & & & \\
\hline & 2 & 1 & 7 & כ. & 25 & & & & & & \\
\hline & 2 & 1 & 8 & 15 & 25 & & & & & & \\
\hline 0.5 & 2 & 1 & 9 & & & & & & & & \\
\hline & 2 & & & & & & & & & & \\
\hline & 2 & 1 & 5 & & & & & & & & \\
\hline 5 & 2 & 1 & 5 & 17 & 25 & & & & & & \\
\hline & 2 & 1 & 5 & 18 & & & & & & & \\
\hline 0.5 & 2 & 1 & 5 & 19 & 25 & & & 391 & 4892 & 4050 & 1 \\
\hline & 2 & 1 & 5 & 15 & & & & & & & \\
\hline 0. & 2 & 1 & 5 & 15 & 2 & & & & & & \\
\hline & 2 & 1 & 5 & 15 & 27 & & & & & & \\
\hline & 2 & 1 & 5 & 15 & 2 & & & & & & \\
\hline & & & 5 & 15 & 20 & & & & & & \\
\hline
\end{tabular}
node, second node and third node are increasing when other parameters remain fixed. When the parameter $(\alpha)$ varies from 1 to 3 , the throughput of the first node, second node and third node are increasing when other parameters remain fixed.

Table 1

Values of Utilization and Throughput of the communication
Network with Dynamic Bandwidth Allocation and Non Homogeneous Process arrivals

*= Seconds, $\$=$ Multiples of 10,000 Packets/sec 


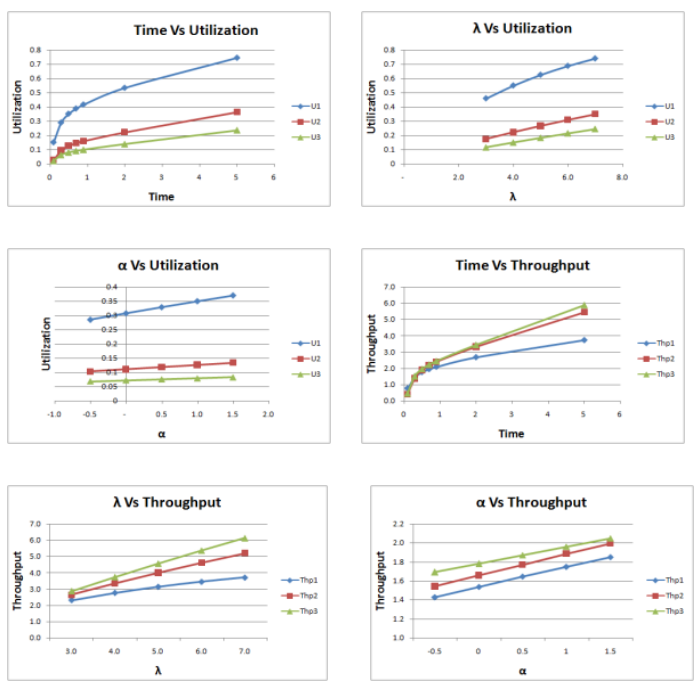

Figure 1 : The relationship between Utilization and Throughput and various other parameters

When the transmission rate $\left(\mu_{1}\right)$ varies from $5 \times 10^{4}$ packets/sec to $9 \times 10^{4}$ packets/sec, the throughput of the first, second and the third node are increasing when other parameters remain fixed. Similarly the transmission rate $\left(\mu_{2}\right)$ varies from $15 \times 10^{4}$ packets/sec to $19 \times 10^{4}$ packets/sec, the throughput of the first node remains constant, the second node and the third node are increasing when other parameters remain fixed. As the transmission rate $\left(\mu_{3}\right)$ varies from $25 \times 10^{4}$ packets/sec to $29 \times 10^{4}$ packets/sec, the throughput of the first and second nodes remains constant and for the third node, it is increasing when other parameters remain fixed.

From equations $(3.8),(3,10),(3.12)$ and (3.18), (3.20), (3.22), the mean number of packets in the buffers and in the network, mean delays in transmission of three transmitters are computed for different values of $\mathrm{t}, \lambda, \alpha, \mu_{1}, \mu_{2}, \mu_{3}$ and presented in Table 2 . The relationship between the parameters and the performance measure are shown in the Figure 2.

It is observed that when time $t=0.1$ seconds, the number of packets in the first buffer is 1616 packets, after 0.3 seconds it rapidly increases to 3396 packets. After of 0.7 seconds, it reaches 4891 packets and thereafter there is steady increase in the content of the first buffer for fixed values of other parameters $(2,1,5,15,25)$ for $\left(\lambda, \alpha, \mu_{1}, \mu_{2}, \mu_{3}\right)$. It is observed that as time $(\mathrm{t})$ varies from 0.1 second to 5 seconds, the average content in second and third buffers and in the network is increasing when other parameters are fixed.

When the parameter $(\lambda)$ varies from $3 \times 10^{4}$ packets $/ \mathrm{sec}$ to $7 \times 10^{4}$ packets/sec, the average content of the first, second and third buffers and in the network are increasing when other parameters remain fixed. The parameter $(\alpha)$ varies from 1 to 3 , the average number of packets in the first, second and third buffers and in the network are increasing when other parameters remain fixed.

When the transmission parameter $\left(\mu_{1}\right)$ varies from $5 \times 10^{4}$ packets/sec to $9 \times 10^{4}$ packets/sec, the average content of the first buffer and in the network are decreasing and the mean number of packets in the second and third buffers are increasing when other parameters remain fixed.

\section{Table 2}

Values of mean number of packets and mean delay of the communication network with DBA and NHP Arrivals

\begin{tabular}{|c|c|c|c|c|c|c|c|c|c|c|c|}
\hline & $\lambda^{5}$ & $\alpha$ & & $\mu_{2}^{s}$ & $\mu_{3}^{s}$ & $\mathrm{~L}_{1}(\mathrm{t})$ & $\mathrm{L}_{2}(\mathrm{t})$ & (t) & $W_{1}(t)$ & $W_{2}(t)$ & \\
\hline .1 & 2 & 1 & 5 & 15 & 25 & 0.16165 & 0.02743 & 0.01862 & 0.21660 & 0.06759 & 0.040 \\
\hline & & 1 & 5 & 15 & 25 & 33967 & 09611 & & 25Jo & & \\
\hline & 2 & 1 & 5 & 15 & 25 & 45 & 3415 & & & & \\
\hline & & 1 & 5 & 15 & 25 & .48913 & 5679 & 09347 & 25288 & 203 & 19 \\
\hline & & 1 & 5 & 15 & 25 & 53600 & 17356 & & & & \\
\hline & & 1 & 5 & 15 & 25 & 75998 & & & & & 303 \\
\hline 0 & & 1 & 5 & 15 & 25 & 1.36000 & 44889 & 0.26773 & 36592 & & \\
\hline & & & 5 & 15 & 5 & & & & & & \\
\hline 5 & 4 & 1 & 5 & 15 & 25 & 0.79762 & 25110 & 145 & 29025 & & \\
\hline 5 & 5 & 1 & 5 & 15 & 25 & 0.98120 & 30958 & & & & \\
\hline 5 & 6 & 1 & 5 & 15 & 25 & 1.16478 & 0.36806 & & & & \\
\hline .5 & 7 & 1 & 5 & 15 & 25 & 1.34836 & .42653 & 0.28129 & 0.36426 & & 589 \\
\hline 5 & & 0.5 & 5 & 5 & 5 & & & & & & \\
\hline .5 & 2 & 0 & 5 & 15 & 25 & 0.36717 & 0.11695 & 0.0 & & & 149 \\
\hline 5 & 2 & 0.5 & 5 & 15 & 25 & 0.39881 & & & & & \\
\hline 5 & 2 & 1 & 5 & 15 & 25 & & & & & & \\
\hline .5 & 2 & 1.5 & 5 & 15 & 25 & 0.46209 & 0.14275 & 0.08544 & 0.24976 & 0.0 & 173 \\
\hline 5 & & 1 & J & & & & & & & & \\
\hline .5 & & 1 & 6 & 15 & 5 & 37368 & 02 & & & & \\
\hline .5 & & 1 & 7 & 15 & 25 & 0.32872 & 14575 & 688 & 62 & & 176 \\
\hline & & 1 & 8 & 15 & 25 & & & & & & \\
\hline .5 & 1 & 1 & 9 & 15 & 25 & 0.26310 & 0.15142 & 0.08959 & 0.12637 & 0.07184 & 4182 \\
\hline 5 & & 1 & 5 & 5 & 25 & & & & & & \\
\hline .5 & & 1 & 5 & & 25 & & & & & & \\
\hline .5 & 2 & 1 & 5 & 17 & 25 & 0.43045 & 0.11953 & 0.08222 & 0.24 & & 167 \\
\hline & & 1 & 5 & 18 & 25 & & & & & & \\
\hline .5 & 2 & 1 & 5 & 19 & 25 & 0.43045 & 0.10773 & 0.08272 & 0.24612 & 0.05552 & 0.0 \\
\hline כ & 2 & 1 & 5 & 15 & 25 & & 15 & & & & \\
\hline .5 & & 1 & 5 & 15 & 26 & & & & & & \\
\hline .5 & 2 & 1 & 5 & 15 & 27 & 0.43045 & 0.13415 & 0.07574 & 0.24 & & 0.0 \\
\hline T & 2 & 1 & 5 & 15 & 28 & | & & & & & \\
\hline .5 & 2 & 1 & 5 & 15 & & 43045 & 13415 & 0 & 024612 & & 0.03572 \\
\hline
\end{tabular}

$*=$ Seconds, $\$=$ Multiples of 10,000 Packets/sec

When the transmission parameter $\left(\mu_{2}\right)$ varies from $15 \times 10^{4}$ packets/sec to $19 \times 10^{4}$ packets/sec, the average content of the first buffer remains constant and the average content of second buffer and in the network are decreasing and in the third buffer the content is increasing when other parameters remain fixed.

Similarly the transmission parameter $\left(\mu_{3}\right)$ varies from $25 \times 10^{4}$ packets/sec to $29 \times 10^{4}$ packets $/ \mathrm{sec}$, the average content of the first and second buffers remain constant and the average content of the third buffer and in the network are decreasing when other parameters remain fixed.

It is observed that as the time (t) and the parameter $(\lambda)$ are increasing, the mean delay in buffers is increasing for fixed values of the other parameters. It is also observed that as the parameter $(\alpha)$ varies the mean delay in all buffers are increasing from fixed values of other parameters. As the transmission rate $\left(\mu_{1}\right)$ increases, the mean delay in the first buffer decreases and 
the mean delay in the second and third buffers increase when the other parameters remain fixed. Similarly, the transmission rate $\left(\mu_{2}\right)$ increases the mean delay in the first buffer remains constant and the mean delay in the second buffer decreases and the mean delay in the third buffer increases when other parameter remains fixed. Similarly, the transmission rate $\left(\mu_{3}\right)$ increases the mean delay in the first and second buffers remain constant and the mean delay in the third buffer decreases when other parameter remains fixed.

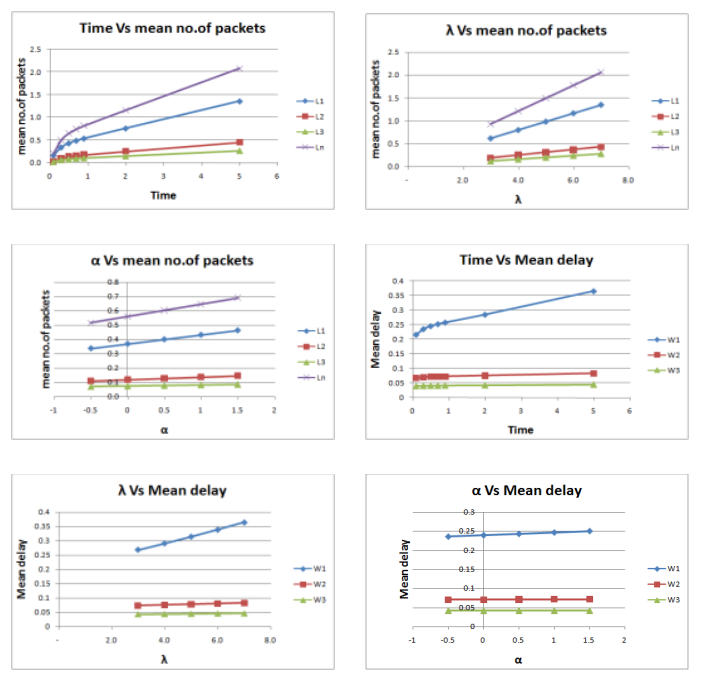

Figure 2 : The relationship between mean no. of packets, mean delay and various parameters

From this analysis it is observed that the dynamic bandwidth allocation strategy has a significant influence on all performance measures of the network. It is further observed that the performance measures are highly sensitive towards smaller values of time. Hence, it is optimal to consider dynamic bandwidth allocation under and non-homogeneous Poisson arrivals and evaluate the performance of the network under transient conditions. It is also to be observed that the congestion in buffers and delays in transmission got reduced to a minimum level by adopting dynamic bandwidth allocation. This phenomenon has a vital bearing on quality of transmission (service).

\section{SENSIVITY ANALYSIS}

Sensitivity analysis of the model is performed with respect to the effect of changes in the parameters $t, \lambda, \alpha, \mu_{1}, \mu_{2}, \mu_{3}$ on the mean number of packets, the utilization of transmitters, the mean delay and the throughput of the first, second and third nodes.

The following data has been considered for the sensitivity analysis, $\mathrm{t}=0.5 \mathrm{sec}, \lambda=2 \times 10^{4}$ packets $/ \mathrm{sec}, \alpha=1, \mu_{1}=5 \times 10^{4}$ packets $/ \mathrm{sec}, \mu_{2}=15 \times 10^{4}$ packets $/ \mathrm{sec}$, and $\mu_{3}=25 \times 10^{4}$ packets $/ \mathrm{sec}$.

The mean number of packets, the utilization of nodes, the mean delay, and the throughput of the first, second and third transmitters are computed with variation of $-15 \%,-10 \%,-5 \%$, $0 \%,+5 \%,+10 \%$ and $+15 \%$ on the model parameters and presented in table 3 .

The performance measures are highly affected by the changes in the values of time $(t)$ and arrival parameters $(\lambda)$ and $(\alpha)$. As $t$ increases from $-15 \%$ to $+15 \%$ the average number of packets in the three buffers and in the total network increase along with the average delay in buffers, the utilization and throughput of the three nodes. As the parameter $(\lambda)$ increases from $-15 \%$ to $+15 \%$ the average number of packets in the three buffers and in the network are increasing along with the average delay, the utilization and the throughput of nodes. Similarly, for the parameter $(\alpha)$, the utilization and the throughput are increasing when it increases. Overall analysis of the parameters reflects that the dynamic bandwidth allocation strategy for congestion control tremendously reduce the delay in transmission and improve the quality of service by reducing burstness in buffers.

\section{Table 3}

Sensitivity Analysis of the Model

\begin{tabular}{|c|c|c|c|c|c|c|c|c|}
\hline \multirow{2}{*}{$\begin{array}{l}\text { Param } \\
\text { eter }\end{array}$} & \multirow{2}{*}{$\begin{array}{l}\text { Perfor } \\
\text { mance } \\
\text { Measur }\end{array}$} & \multicolumn{7}{|c|}{$\%$ change in Parameter } \\
\hline & & -15 & -10 & -5 & 0 & 5 & 10 & 15 \\
\hline \multirow{12}{*}{$t=0.5$} & $L_{1}(t)$ & 0.402004 & 0.412056 & 0.421515 & 0.430449 & 0.438922 & 0.446986 & 0.454690 \\
\hline & $L_{2}(t)$ & 0.122501 & 0.126659 & 0.130531 & 0.134149 & 0.137541 & 0.140732 & 0.143746 \\
\hline & $L_{3}(t)$ & 0.075735 & 0.077801 & 0.079738 & 0.081564 & 0.083291 & 0.084933 & 0.086498 \\
\hline & $\mathrm{U}_{1}(\mathrm{t})$ & 0.331022 & 0.337713 & 0.343948 & 0.349783 & 0.355269 & 0.360447 & 0.365355 \\
\hline & $\mathrm{U}_{2}(\mathrm{t})$ & 0.116096 & 0.120247 & 0.124115 & 0.127730 & 0.131119 & 0.134309 & 0.137321 \\
\hline & $\mathrm{U}_{3}(\mathrm{t})$ & 0.073493 & 0.075558 & 0.077495 & 0.079321 & 0.081048 & 0.082690 & 0.084255 \\
\hline & $W_{1}(t)$ & 0.242887 & 0.244027 & 0.245104 & 0.246124 & 0.247093 & 0.248018 & 0.248903 \\
\hline & $W_{2}(t)$ & 0.070833 & 0.070978 & 0.071112 & 0.071238 & 0.071356 & 0.071468 & 0.071573 \\
\hline & $W_{3}(t)$ & 0.041534 & 0.041576 & 0.041616 & 0.041653 & 0.041689 & 0.041723 & 0.041755 \\
\hline & $\mathrm{Thp}_{1}(\mathrm{t}$ & 1.655110 & 1.688565 & 1.719739 & 1.748916 & 1.776344 & 1.802236 & 1.826777 \\
\hline & $\operatorname{Thp}_{2}(\mathrm{t}$ & 1.729421 & 1.784491 & 1.835567 & 1.883107 & 1.927519 & 1.969169 & 2.008379 \\
\hline & $\mathrm{Thp}_{3}(\mathrm{t}$ & 1.823463 & 1.871280 & 1.916050 & 1.958156 & 1.997926 & 2.035646 & 2.071564 \\
\hline \multirow{12}{*}{$\lambda=2$} & $\mathrm{~L}_{1}(\mathrm{t})$ & 0.375375 & 0.393733 & 0.412091 & 0.430449 & 0.448808 & 0.467166 & 0.485524 \\
\hline & $L_{2}(t)$ & 0.116606 & 0.122454 & 0.128302 & 0.134149 & 0.139997 & 0.145845 & 0.151692 \\
\hline & $L_{3}(t)$ & 0.069581 & 0.073575 & 0.077569 & 0.081564 & 0.085558 & 0.089553 & 0.093547 \\
\hline & $\mathrm{U}_{1}(\mathrm{t})$ & 0.312968 & 0.325466 & 0.337736 & 0.349783 & 0.361611 & 0.373224 & 0.384626 \\
\hline & $\mathrm{U}_{2}(\mathrm{t})$ & 0.112188 & 0.117408 & 0.122589 & 0.127730 & 0.132831 & 0.137893 & 0.142916 \\
\hline & $\mathrm{U}_{3}(\mathrm{t})$ & 0.068056 & 0.071826 & 0.075581 & 0.079321 & 0.083046 & 0.086756 & 0.090451 \\
\hline & $W_{1}(t)$ & 0.239880 & 0.241950 & 0.244031 & 0.246124 & 0.248227 & 0.250341 & 0.252466 \\
\hline & $W_{2}(t)$ & 0.070629 & 0.070832 & 0.071035 & 0.071238 & 0.071442 & 0.071646 & 0.071851 \\
\hline & $W_{3}(t)$ & 0.041408 & 0.041490 & 0.041571 & 0.041653 & 0.041736 & 0.041818 & 0.041900 \\
\hline & $\mathrm{Thp}_{1}(\mathrm{t}$ & 1.564840 & 1.627329 & 1.688680 & 1.748916 & 1.808056 & 1.866120 & 1.923128 \\
\hline & $\mathrm{Thp}_{2}(\mathrm{t}$ & 1.650967 & 1.728800 & 1.806179 & 1.883107 & 1.959586 & 2.035619 & 2.111209 \\
\hline & $\operatorname{Thp}_{3}(\mathrm{t}$ & 1.680375 & 1.773339 & 1.865932 & 1.958156 & 2.050012 & 2.141502 & 2.232627 \\
\hline \multirow{12}{*}{$\alpha=1$} & $\mathrm{~L}_{1}(\mathrm{t})$ & 0.420957 & 0.424121 & 0.427285 & 0.430449 & 0.433614 & 0.436778 & 0.439942 \\
\hline & $L_{2}(t)$ & 0.131570 & 0.132430 & 0.133289 & 0.134149 & 0.135009 & 0.135869 & 0.136729 \\
\hline & $L_{3}(t)$ & 0.080402 & 0.080789 & 0.081177 & 0.081564 & 0.081951 & 0.082339 & 0.082726 \\
\hline & $\mathrm{U}_{1}(\mathrm{t})$ & 0.343582 & 0.345655 & 0.347723 & 0.349783 & 0.351837 & 0.353885 & 0.355926 \\
\hline & $\mathrm{U}_{2}(\mathrm{t})$ & 0.124856 & 0.125815 & 0.126773 & 0.127730 & 0.128686 & 0.129642 & 0.130597 \\
\hline & $\mathrm{U}_{3}(\mathrm{t})$ & 0.078037 & 0.078465 & 0.078893 & 0.079321 & 0.079748 & 0.080175 & 0.080602 \\
\hline & $W_{1}(t)$ & 0.245040 & 0.245401 & 0.245762 & 0.246124 & 0.246485 & 0.246847 & 0.247210 \\
\hline & $W_{2}(t)$ & 0.071148 & 0.071178 & 0.071208 & 0.071238 & 0.071268 & 0.071298 & 0.071328 \\
\hline & $W_{3}(t)$ & 0.041630 & 0.041638 & 0.041645 & 0.041653 & 0.041661 & 0.041669 & 0.041677 \\
\hline & $\operatorname{Thp}_{1}(\mathrm{t}$ & 1.717908 & 1.728277 & 1.738613 & 1.748916 & 1.759187 & 1.769425 & 1.779631 \\
\hline & $\operatorname{Thp}_{2}(\mathrm{t}$ & 1.849229 & 1.860531 & 1.871824 & 1.883107 & 1.894380 & 1.905643 & 1.916897 \\
\hline & $\mathrm{Thp}_{3}(\mathrm{t}$ & 1.931361 & 1.940296 & 1.949227 & 1.958156 & 1.967080 & 1.976002 & 1.984920 \\
\hline
\end{tabular}

\section{COMPARATIVE STUDY}

To study the effect of non homogeneous Poisson arrival assumption on the communication network a comparative study 
between the performance measures of the network models with non homogeneous Poisson arrivals and Poisson arrivals is performed. The performance measures of both models are computed with fixed values of the parameters $\lambda, \alpha, \mu_{1}, \mu_{2}, \mu_{3}$ and different values of $t=0.3,0.5$ seconds and presented in table 4 .

As $t$ increases the percentage variation of performance measures between the models is increasing. For the model with nonhomogeneous Poisson arrivals with dynamic bandwidth allocation has more utilization compared to that of the model with Poisson arrivals with dynamic bandwidth allocation. From this analysis it is observed that the assumption of nonhomogeneous Poisson arrivals have a significant influence on all the performance measures of the network. This model also includes the two node tandem communication network model when $\mu_{3}$ is zero.

Table 4

Comparative study of models with NHP and HP arrivals

\begin{tabular}{|c|c|c|c|c|c|}
\hline Time & Paramete & Model & Model & Differenc & $\%$ \\
\hline \multirow[t]{12}{*}{$t=0.3$} & $L_{1}(t)$ & 0.339673 & 0.310748 & 0.028925 & 4.45 \\
\hline & $L_{2}(t)$ & 0.096108 & 0.089448 & 0.006660 & 3.59 \\
\hline & $L_{3}(t)$ & 0.062723 & 0.062693 & 0.000030 & 0.02 \\
\hline & $\mathrm{U}_{1}(\mathrm{t})$ & 0.287997 & 0.267101 & 0.020896 & 3.76 \\
\hline & $U_{2}(t)$ & 0.089821 & 0.081125 & 0.008696 & 5.09 \\
\hline & $U_{3}(t)$ & 0.060483 & 0.059580 & 0.000902 & 0.75 \\
\hline & $W_{1}(t)$ & 0.235887 & 0.232682 & 0.003205 & 0.68 \\
\hline & $W_{2}(t)$ & 0.069922 & 0.069693 & 0.000229 & 0.16 \\
\hline & $W_{3}(t)$ & 0.041268 & 0.041267 & 0.000001 & 0.01 \\
\hline & $\operatorname{Thp}_{1}(\mathrm{t})$ & 1.439985 & 1.335507 & 0.104478 & 3.76 \\
\hline & $\operatorname{Thp}_{2}(\mathrm{t})$ & 1.374512 & 1.283461 & 0.091051 & 3.43 \\
\hline & $\operatorname{Thp}_{3}(\mathrm{t})$ & 1.519912 & 1.519210 & 0.000702 & 0.02 \\
\hline \multirow[t]{12}{*}{$t=0.5$} & $\mathrm{~L}_{1}(\mathrm{t})$ & 0.430449 & 0.367166 & 0.063283 & 7.93 \\
\hline & $L_{2}(t)$ & 0.134149 & 0.116953 & 0.017196 & 6.85 \\
\hline & $L_{3}(t)$ & 0.081564 & 0.073816 & 0.007748 & 4.99 \\
\hline & $\mathrm{U}_{1}(\mathrm{t})$ & 0.349783 & 0.307305 & 0.042478 & 6.46 \\
\hline & $U_{2}(t)$ & 0.127730 & 0.108456 & 0.019274 & 8.16 \\
\hline & $\mathrm{U}_{3}(\mathrm{t})$ & 0.079321 & 0.070700 & 0.008621 & 5.75 \\
\hline & $W_{1}(t)$ & 0.246124 & 0.238958 & 0.007165 & 1.48 \\
\hline & $W_{2}(t)$ & 0.071238 & 0.070641 & 0.000597 & 0.42 \\
\hline & $W_{3}(t)$ & 0.041653 & 0.041494 & 0.000159 & 0.19 \\
\hline & $\operatorname{Thp}_{1}(\mathrm{t})$ & 1.748916 & 1.536527 & 0.212389 & 6.46 \\
\hline & $\operatorname{Thp}_{2}(\mathrm{t})$ & 1.883107 & 1.655598 & 0.227509 & 6.43 \\
\hline & $\operatorname{Thp}_{3}(\mathrm{t})$ & 1.958156 & 1.778932 & 0.179224 & 4.80 \\
\hline
\end{tabular}

\section{CONCLUSION}

This paper deals with a novel and new approach on performance evaluation of tandem communication networks by characterizing that the arrival of packets with a non homogeneous Poisson process and utilizing the dynamic bandwidth allocation strategy of transmission. The non homogeneous Poisson arrivals can model the time dependent nature of bursty arrivals. Using the difference-differential equation the behaviour of network is analyzed by deriving explicit expressions for the network performance measures like average number of packets in each buffer, the mean delay in buffer, throughput of nodes, and utilization of the transmitters. The sensitivity analysis of network reveals that the dynamic Bandwidth allocation strategy can reduce the congestion in buffers and mean delay in transmission. A comparative study of the proposed model with that of Poisson arrivals indicate that the proposed model outperform in predicting the performance measures more accurately under heavy (peak) traffic conditions then other models. This model also includes some of the earlier models as particular cases when the time dependent arrival parameter $\alpha=0$. This model is much useful in controlling and monitoring the communication network more effectively and efficiently.

\section{REFERENCES}

[1] Dinda P.A.,(2006), Design, Implementation and performance of an Extensible toolkit in resource prediction in Distributed systems, IEEE Transactions on Parallel and Distributed systems, vol.17, no.2, pp.160-173.

[2] Huang, Scott C.H, Chang, Shih Yu,(2010), Analysis and design of a novel randomized Broadcast algorithm for scalable Wireless Networks in the Interference Channels, IEEE Transactions on Wireless Communications, Vol.9, No.7, pp.2206-2215

[3] Javadi B., J. H. Abawajy and M. K. Akban (2007), A comprehensive Analytical model of interconnection Network in large scale cluster systems, concurrency and computation practice and Experience, Vol.20, No.1, pp.7597.

[4] Kin K Leung (2002), Load dependent service queues with application to congestion control in broadband Networks, performance evaluation, vol. 50, issue 1-4, pp. 27-40.

[5] Kleinrock L., (1976), Queuing system, vol.II, Computer Applications, wiley publications, Newyork.

[6] Nageswara Rao K., K. Srinivas Rao, P Srinivasa Rao (2010), A tandem communication Network with Dynamic Bandwidth Allocation and modified phase type transmission having Bulk arrivals, International Journal of Computer science Issues, vol.7, no.3, pp 18-26.

[7] Padmavathi G., K. Srinivas Rao, KVVS Reddy (2009), Performance Evaluation of parallel and series communication Network with Dynamic Bandwidth Allocation, CIIT International Journal of Networking and communication Engineering Vol.1, No. 7, pp 410-421.

[8] Srinivasa Rao K., Vasanta M.R., and Vijaya kumar C.V.R.S.,(2000), On an interdependent communication network, ,Opsearch vol.37, no.2, pp 134-143.

[9] Srinivasa Rao, K., Prasad Reddy, P.V.G.D. and Suresh Varma,P. (2006), Interdependent Communication Network with bulk arrivals, International Journal of Management and Systesms,Vol.22, No.3, pp 221-234.

[10] Sriram K.(1993), Methodologies for bandwidth allocation, transmission scheduling and congestion control avoidance in broadband ATM Networks, computer Network , ISDN system, j.26, pp. 43-59

[11] Suresh Varma P., K. Srinivas Rao (2007), A Communication Network with load dependent Transmission, International Journal of Mathematical Sciences, vol.6, no.2, pp 199-210.

[12] Yulei Wu, Geyong Min, Keqin Li and Bahman Javadi (2009), Performance Analysis of communication Network in multi-cluster systems under bursty traffic with 
communication locality, Proceedings of IEEE "GLOBECOM", pp.1-6

[13] Yukuo Hayashida (1993), Throughput analysis of tandem type go-back NARQ scheme for satellite communications, IEEE Transactions on communication, vol.41, pp 1517-1524. 